\title{
Rapport annuel du président des délégués aux questions des assistantes médicales
}

Emil Schalch

Correspondance:

Dr Emil Schalch

Président des délégués

cantonaux aux questions des assistantes médicales

Elfenstrasse 18

CH-3000 Berne 15

\section{Réforme de l'ordonnance sur la formation des assistantes médicales}

L'Ordonnance sur la formation professionnelle initiale d'assistante médicale et le plan de formation sont entrés en vigueur le $1^{\text {er }}$ janvier 2010. Ces nouvelles bases de formation seront progressivement introduites dès la rentrée scolaire d'août 2010. Les apprenantes qui commencent maintenant leur $2^{\mathrm{e}}$ ou leur $3^{\mathrm{e}}$ année de formation la termineront d'après l'ancien règlement. Tous les contenus d'apprentissage et les plans de formation coordonnés se trouvent sur le site www.mpaschweiz.ch.

La rédaction en allemand de l'Aide-mémoire pour les assistantes médicales, souhaité de toutes parts, est achevée; ce manuel est disponible pour la nouvelle année scolaire 2010. La version française est prévue pour cet automne.

La Documentation pour les assistantes médicales (autrefois journal de travail) a été remaniée par l'Association suisse des assistantes médicales également pour la rentrée d'août 2010; elle est disponible en français, en allemand et en italien.

La «Commission suisse pour le développement professionnel et la qualité pour les assistantes médicales» s'est constituée dans le cadre d'une première séance tenue en janvier 2010. Le corps médical est re-

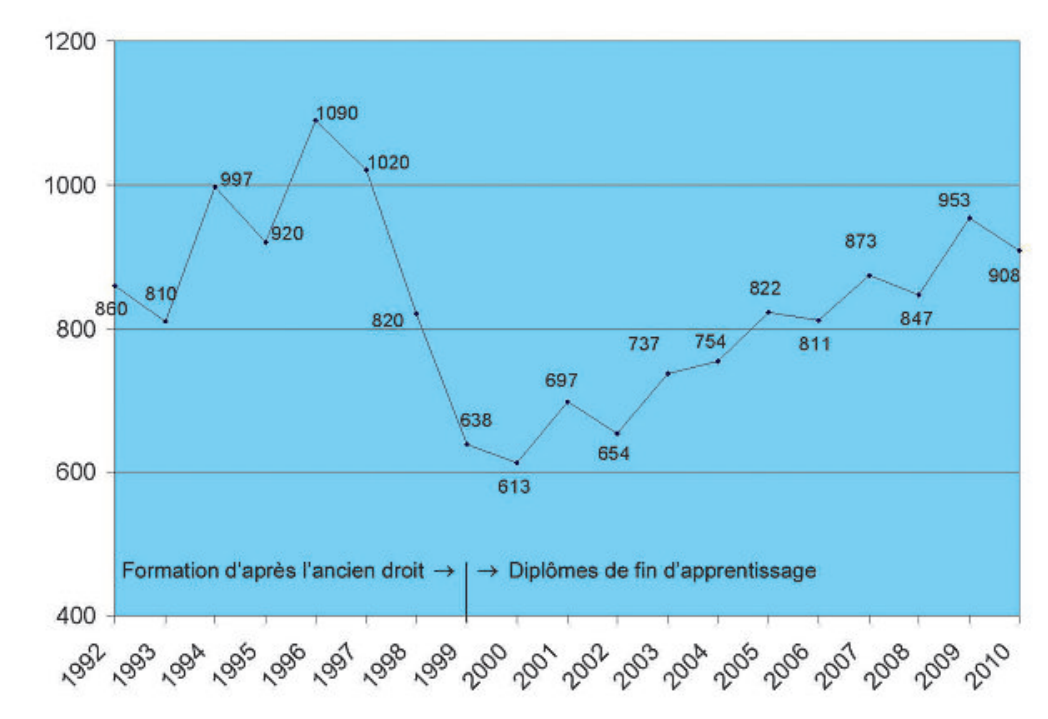

Statistique des assistantes médicales diplomées depuis 1992. présenté par le Dr Peter Tschudi (président), le Dr Emil Schalch (vice-président) et le Dr Daniel Wildhaber. Le secrétariat de la Commission est géré par le service MPA (assistantes médicales) du Secrétariat général de la FMH. Cette Commission réunit des représentants des associations d'assistantes médicales, de l'Office fédéral de la formation professionnelle et de la technologie (OFFT), des offices cantonaux, de l'Office fédéral de la santé publique (OFSP) et des écoles. Elle a notamment pour tâches d'adapter régulièrement, au moins tous les 5 ans, le plan de formation aux développements économiques, technologiques et didactiques. En outre, elle peut proposer à l'OFFT des modifications de l'Ordonnance et elle adopte les conditions de réussite pour la validation des prestations de formation. Pour des raisons d'efficacité, il est d'ailleurs prévu de lui confier les tâches de la Commission de surveillance des cours interentreprises.

\section{Formation des assistantes médicales et cours de maîtres d'apprentissage}

En 2010, le nombre d'assistantes médicales diplômées s'est élevé à 908. Nous avons toujours besoin de nouvelles places d'apprentissage. Je saisis l'occasion pour encourager tous les médecins qui ne forment pas encore d'apprenantes à le faire, car cette activité est loin de représenter uniquement une charge de travail supplémentaire, elle permet aussi d'assumer une belle tâche et de faire en sorte que nous continuions à disposer de suffisamment d'assistantes médicales bien formées. Les maîtres d'apprentissage ont désormais à leur disposition un outil précieux, en l'occurrence l'aide-mémoire pour assistantes médicales mentionné en début du présent rapport.

La participation aux cours de maîtres d'apprentissage est constante. Deux cours destinés aux médecins ont à nouveau pu être organisés durant l'exercice écoulé.

Nous remercions cordialement les médecins qui forment régulièrement de jeunes assistantes médicales.

\section{Formation complémentaire des assistantes médicales}

Les travaux concernant la formation complémentaire des assistantes médicales sont effectués dans le cadre de l'organisation du monde du travail (ORTRA), Formation des assistantes médicales. La FMH est représentée au plus haut niveau par le Dr Schalch, président de 
l'ORTRA, et le Dr Tschudi, président de la Commission Assurance-qualité. Deux certificats fédéraux d'aptitude professionnelle sont prévus: l'un axé sur l'aspect administratif, en vue du titre de responsable de cabinet médical et l'autre axé sur l'aspect clinique et intitulé «Chronic Care Management». Le site internet de l'ORTRA www.odamed.ch donne des informations sur ces travaux au fur et à mesure de leur avancement.

\section{Fonds de formation professionnelle}

Les travaux relatifs à la création d'un fonds de formation professionnelle sont restés bloqués durant la période passée sous revue. Le rapprochement des points de vue de l'Association suisse des assistantes médicales/FMH et de la Fédération suisse des associations d'assistantes médicales (FSAAM) n'a pas eu lieu, alors qu'il permettrait d'achever rapidement ces travaux.

\section{Commission Examen final d'apprentissage}

Cette commission a effectué une nouvelle fois un travail important sous la direction du Dr Peter Tschudi. Les documents d'examens ont à nouveau pu être transmis aux offices concernés en temps voulu. A l'occasion de la séance d'évaluation du mois d'août, le Dr Peter Tschudi a remis son mandat de président de la Commission de surveillance au Dr Peter Imbach. Nous lui exprimons notre vive gratitude pour son engagement infatigable durant de longues années, tout d'abord en qualité de chef de groupe puis comme successeur de Mme le Dr Verena Gantner.

\section{Saisie de la totalité des coûts pour les cours interentreprises par le Réseau des employeurs pour la formation professionnelle (SQUF)}

Le réseau des employeurs pour la formation professionnelle (SQUF) saisit à intervalles réguliers la totalité des coûts pour les cours interentreprises, afin que la contribution cantonale par journée de cours interentreprises et par apprenante puisse être fixée sur cette base. Une telle saisie a eu lieu la première fois en 2006. La moyenne suisse doit être calculée pour les professions disposant de différents lieux de formation interentreprises. La Conférence suisse des offices de formation professionnelle (CSFP) a approuvé une méthode de calcul qui prend en compte les chiffres à venir et non ceux présents, car il faut s'attendre à des coûts plus élevés avec la révision de la formation. Finalement, on est parvenu au résultat de 212 francs par journée de cours interentreprises et par apprenante.

\section{Validation des prestations de formation}

Ce point se trouve depuis longtemps sur la liste des affaires en suspens des responsables MPA de la FMH comme aussi sur celle de l'ORTRA Formation des assistantes médicales.
Les compétences professionnelles pratiques sont acquises de différentes manières. Outre la formation formelle, il faut aussi prendre en considération le quotidien professionnel et extra-professionnel car ces compétences s'acquièrent avec l'expérience. La validation des prestations de formation permet de saisir des prestations très différentes dans une procédure structurée, d'attester les compétences professionnelles pratiques et de parvenir à un certificat de fin d'apprentissage formel.

L'OFFT exige de l'ORTRA d'élaborer les documents nécessaires: profil de qualification et conditions de réussite sur la base du plan de formation. Ces documents doivent être adoptés par la commission «Développement professionnel et qualité» (cf. règlement d'organisation art. 5) regroupant l'ensemble des partenaires concernés, laquelle soumet ensuite une proposition à l'OFFT.

Durant l'année écoulée, les responsables en charge du dossier des assistantes médicales de la FMH n'ont rien entrepris dans ce dossier mais des activités ont été menées à ce sujet en Suisse romande et ont été présentées à l'assemblée annuelle des délégués aux questions des assistantes médicales à Lucerne.

\section{Contrat type pour assistantes médicales - Adaptations suite à l'intervention du Centre de liaison des associations féminines de Zurich}

Après que le Centre de liaison des associations féminines de Zurich a estimé en automne 2008 que la réglementation des heures supplémentaires était discriminatoire pour les personnes engagées à temps partiel, un compromis a été trouvé l'automne dernier entre les éditeurs communs du contrat-type, compromis qui répond à toutes les préoccupations. Nous avons publié une brève information à ce sujet dans le BMS [1].

\section{Office de conciliation}

Durant la période passée sous revue, aucune demande de conciliation pour la Suisse allemande n'est parvenue au Prof. J. Brühwiler, Dr en droit, et il n'y a eu aucun dossier en suspens.

\section{En conclusion: à moins de le faire soi-même!} Ainsi qu'on a pu le lire ces derniers temps dans le BMS et la presse quotidienne, il est prévu d'attribuer un rôle plus important aux assistantes médicales lors du repositionnement des soins de premier recours, afin de permettre à la médecine de famille de relever les défis du futur. De nombreuses questions restent cependant ouvertes et nécessitent encore d'être traitées. J'invite donc tous les médecins à s'engager en faveur d'une bonne formation professionnelle initiale et complémentaire des assistantes médicales. Cela en vaut la peine! et explications relatives au contrat de travail Assistantes médicales. Bull Med Suisses. 2009;90(48):1865. 